\title{
Protective mechanisms of triptorelin against tripterygium glycoside-induced ovarian dysfunction.
}

\author{
Yang-Chun Zhao ${ }^{1}$, Ling Lv ${ }^{1 *}$, Dong-Sheng Li ${ }^{1}$, Jing Zhao', Hao-Ying Wang ${ }^{2}$ \\ ${ }^{1}$ Department of Gynecology and Obstetrics, the Second Affiliated Hospital of Zhejiang Chinese Medical University, \\ Hangzhou, PR China \\ ${ }^{2}$ Second Clinical Medical College, Zhejiang Chinese Medical University, Hangzhou, PR China
}

\begin{abstract}
The aim of this study was to investigate the protective effects of Triptorelin (TRI) on Tripterygium Glycoside-induced Ovarian Dysfunction (TG-OD). Thirty mice with normal estrous cycles were randomly divided into three groups $(\mathrm{n}=10$ ): the control group (group $\mathrm{A}$ ) was orally administered $0.35 \mathrm{ml}$ of saline daily; the TH group (group B) was orally administered $0.35 \mathrm{ml}$ of TG solution from the $8^{\text {th }} \mathrm{d}$ for 10 w continuously; the TG+TRI group (group C) was subcutaneously injected with $0.1 \mathrm{mg} / \mathrm{kg}$ TRI daily, as well as orally administered $0.35 \mathrm{ml}$ of TG solution from the $8^{\text {th }} \mathrm{d}$ for $10 \mathrm{w}$ continuously. All mice were sacrificed during the 11th week to detect blood anti-Mullerian hormone, follicle-stimulating hormone, luteinizing hormone, and estradiol; calculate the ovarian index; and observe the morphologies of ovarian tissues. Mice in group $\mathrm{C}$ were more flexible, and had increased weight, reduced folliclestimulating hormone and luteinizing hormone, and increased anti-Mullerian hormone and estradiol; additionally, ovarian follicles and mature follicles increased. TG caused gonad and ovarian dysfunction in female mice; TRI exhibited protective effects against TG-induced gonad and ovarian dysfunction in female mice.
\end{abstract}

Keywords: Triptorelin, Tripterygium glycosides, Ovarian function.

Accepted on November 17, 2017

\section{Introduction}

Tripterygium Glycosides (TG) are extracts obtained from a traditional Chinese medicine with immunomodulatory and anti-tumor effects; because the incidence and severity of side effects following TG administration are much lower than those of immunosuppressive agents, TG has been widely used in the clinical treatment of kidney diseases or rheumatism. Rational application of TG may significantly reduce the amount of immunosuppressive drugs required for therapy, thus greatly reducing medical costs and side effects as well as increasing patient compliance. Based on extensive applications and studies of TG, TG was observed to cause damage to the female gonad and ovarian functions [1]; therefore, its application in female patients before and in childbearing age has been extremely limited. It is important to minimize the side effects of TG, which would have important clinical and social effects.

Gonadotropin-releasing hormone agonist (GnRH-a) [2,3] is a synthesized gonadotropin-releasing hormone and is a preferred ovarian function-protecting agent in chemotherapy [4-6], and thus has been widely used in clinical practice [7]. The synthesized analog of GnRH, Triptorelin (TRI), has the same effects as GnRH [8]. Early in the first administration, GnRH agonists completely bind GnRH receptors on the surface of the pituitary gland, stimulating the transient increase of FollicleStimulating Hormone (FSH) and Luteinizing Hormone (LH) (the "ignition" period) [9]. Approximately $15 \mathrm{~d}$ later, the receptors become exhausted and are no longer sensitive to GnRH-a; thus, FSH and LH begin to decrease significantly and ovarian hormones begin to significantly decrease to postmenopausal levels. Therefore, artificial menopause can occur and protect the ovaries.

This study was performed to observe and compare the changes in the estrous cycle and morphologies of ovarian tissues in Kunming mice after administration of TRI and TG; additionally, changes in serum anti-Mullerian hormone (AMH) [10], FSH, LH, and estradiol (E2) were detected to investigate the protective effects of $\mathrm{GnRH}$ against TG-induced ovarian dysfunction in female mice and develop ovarian functionprotective protocols for effective application of TG.

\section{Materials and Methods}

\section{Animals and reagents}

Thirty healthy, female Kunming (KM) mice, 7-8 w old, weighing 32-36 g, and clean-grade, were provided by Shanghai Slaccas Experimental Animal Co., Ltd. (Shanghai, China). 
After 1-2 d adaptive feeding, all mice were subjected to the smear method to collect vaginal exfoliated cells, and those with a normal estrous cycle were selected for the study. This study was carried out in strict accordance with the recommendations in the Guide for the Care and Use of Laboratory Animals of the National Institutes of Health. The animal use protocol has been reviewed and approved by the Institutional Animal Care and Use Committee (IACUC) of Zhejiang Chinese Medical University.

An Enzyme-Linked Immunosorbent Assay (ELISA) kit (Hangzhou Chengwei Biotechnology Co., Ltd., Zhejiang, China) was used to detect AMH, FSH, LH, and E2. Pathological detection reagents included xylene, formaldehyde, 95\% ethanol, hematoxylin and eosin stain, wax, and were provided by the Animal Experimental Center of Zhejiang Chinese Medical University. TG tablets containing $10 \mathrm{mg} /$ tablet, which are typically administered at 2-3 tablets/dose to adults, were orally administered three times per day (Zhejiang DND Pharmaceutical Co., Ltd., Zhejiang, China; State medical Approval No: Z33020422); $1 \mathrm{ml}$ achieve $0.1 \mathrm{mg}$ TRI acetate was injected (calculated as C64H82N18O13). The common dose in adults is $0.5 \mathrm{mg}$ subcutaneous injection once per day (Chengdu Tiantaishan Pharmaceutical Co., Ltd., Chengdu, China; State medical Approval No: H20058648); to prepare TG solution, TG tablets were dissolved in saline to achieve a concentration of $40 \mathrm{mg} / \mathrm{kg}$.

\section{Experimental design}

Thirty qualified KM mice were randomly divided into three groups $(n=10)$. The control group was orally administered 0.35 $\mathrm{ml}$ of saline daily for $11 \mathrm{w}$. The $\mathrm{TH}$ group was orally administered $0.35 \mathrm{ml}$ of TG solution on the $8^{\text {th }} \mathrm{d}$ for $10 \mathrm{w}$ continuously. The TG+TRI group was subcutaneously injected with $0.1 \mathrm{mg} / \mathrm{kg}$ TRI daily for $11 \mathrm{w}$, as well as orally administered $0.35 \mathrm{ml}$ of TG solution on the $8^{\text {th }} \mathrm{d}$ for $10 \mathrm{w}$ continuously.

All mice were sacrificed during w 11 to detect blood AMH, $\mathrm{FSH}, \mathrm{LH}$, and E2, calculate the ovarian index, and observe the morphologies of ovarian tissues. The best protocol was then screened.

\section{General information}

From the first day of the experiment, the general conditions of mice in each group were observed and recorded, including spirit, activity, fur, food intake, water intake, appetite, urine, and stool; their body weights were determined weekly.

The estrous cycle in mouse is approximately 4-5 d, including diestrus, proestrus, estrus, and metestrus. This study used a relatively simple smear method of vaginal exfoliated cells to observe the estrous cycle.

\section{ELISA}

All mice were sacrificed during w 11 and blood samples were obtained using the eyeball enucleation method to detect the serum levels of AMH, FSH, LH, and E2 by ELISA.

\section{Tissue section}

The abdomen of each mouse was then cut open to obtain the ovaries, which were first weighed to determine the wet weight using an electronic analytical balance, followed by calculation of the ovary index=ovary wet weight $(\mathrm{mg}) /$ mouse body weight $(\mathrm{g}) \times 100 \%$. Ovaries were then fixed with $4 \%$ paraformaldehyde, followed by paraffin-embedding, serial slicing, drying, and hematoxylin and eosin staining; the numbers of different-stage ovarian follicles were observed and analysed under a microscope.

Figure 1 shows ovarian tissue slices under the light microscope, including the primordial follicle, primary follicle, secondary follicle, mature follicle, and corpus luteum. To simplify the experiment, the original, primary, and secondary follicles were referred to as follicles.

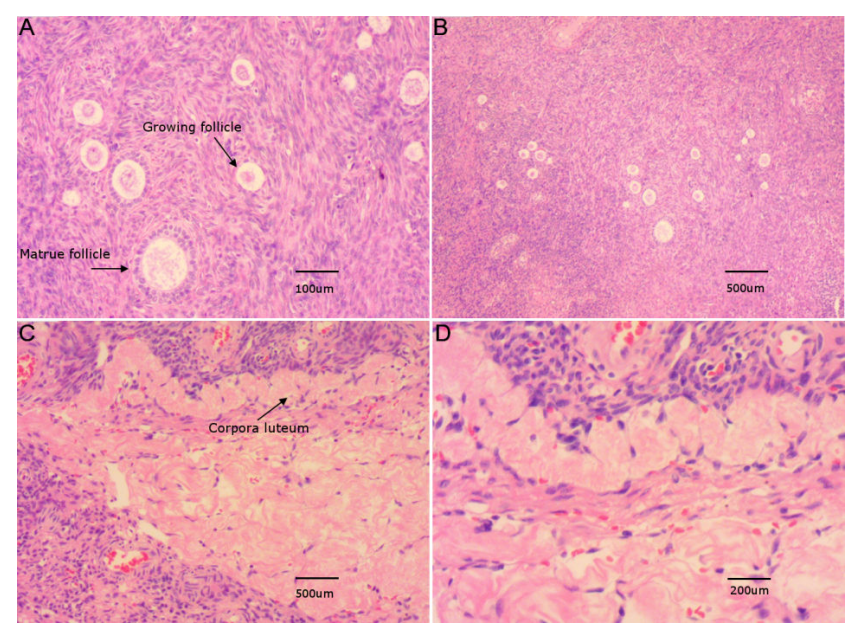

Figure 1. Ovarian tissue sections. A. Under the microscope of mature and growing follicles (field diameter $1 \mathrm{~mm}$, the eyepiece magnification 10, lens magnification 20). B. Under the microscope of mature and growing follicles (field diameter $5 \mathrm{~mm}$, the eyepiece magnification 10 , lens magnification 4). C. Under the microscope of corpora luteum (field diameter $5 \mathrm{~mm}$, the eyepiece magnification 10, lens magnification 4). D. Under the microscope of corpora luteum (field diameter $2 \mathrm{~mm}$, the eyepiece magnification 10, lens magnification 10).

\section{Statistical analysis}

All data were analysed with SPSS statistical software (SPSS, Inc., Chicago, IL, USA). Differences among groups were detected using the Student-Newman-Keuls method. Categorical data were analysed using variance analysis, and the homogeneity test of variance was performed. All results are expressed as the mean \pm standard error of the mean. Statistical significance was accepted at $\mathrm{P}<0.05$. 


\section{Results}

\section{General data}

After one week of feeding, mice in group B showed significantly reduced activities and the amounts of food and water intake gradually reduced; after continuous feeding, the mice exhibited overall edema. However, mice in groups A and $\mathrm{C}$ exhibited better performance with lustrous fur, flexible activities, and increased food and water intake. The performance sorting of the three groups was as follows: group C $>$ group A $>$ group B.

Table 1 shows the results of Analysis of Variance (ANOVA), which revealed no significant difference in the original body weight and body weight $10 \mathrm{w}$ later among different groups ( $\mathrm{P}>0.05)$; however, differences in the ovarian weight and ovarian index among different groups were significant $(\mathrm{P}<0.05)$. Further pairwise comparison showed that the differences in ovarian weight between any two groups were significant $(\mathrm{P}<0.05)$. Ovarian weights differed between groups as follows: group $\mathrm{C}>$ group $\mathrm{A}>$ group $\mathrm{B}$; pairwise comparisons of ovarian index between any two groups also revealed significant differences $(\mathrm{P}<0.05)$, with ovarian index values in the order of group $\mathrm{C}>$ group $\mathrm{A}>$ group $\mathrm{B}$.

\section{Effect of GnRH treatment on estrous cycle}

The estrous cycle is the foundation of ovarian endocrine functions, which may exhibits regular differences under normal ovarian functions and coordination of estrogen and progesterone. After administration of $\mathrm{TG}$ tablets, vaginal smears showed a disturbance in the estrous cycle of mice in group $\mathrm{B}$, indicating that $\mathrm{TG}$ significantly inhibits mouse ovarian endocrine functions. However, mice in group $\mathrm{C}$ exhibited stable estrous cycles, which were extended to 6-7 d.

Table 2 shows the results of ANOVA, which revealed significant differences in the serum levels of AMH, FSH, LH, and E2 in different groups $(\mathrm{P}<0.05)$; further pairwise comparisons showed that the sorting of $\mathrm{AMH}$ was as follows: group $\mathrm{C}>$ group $\mathrm{A}>$ group $\mathrm{B}$; the sorting of $\mathrm{FSH}$ was group $\mathrm{B}>$ group $\mathrm{A}>$ group $\mathrm{C}$; the sorting of $\mathrm{LH}$ was group $\mathrm{B}>$ group $\mathrm{A}>$ group $\mathrm{C}$; the sorting of $\mathrm{E} 2$ was group $\mathrm{C}>$ group $\mathrm{A}>$ group $\mathrm{B}$.

\section{Effect of GnRH treatment on ovarian follicles}

Table 3 shows the results of ANOVA, which revealed significant differences in growing ovarian follicles, mature ovarian follicles, total ovarian follicles, ratio of mature ovarian follicles to total ovarian follicles, and corpora lutea among different groups $(\mathrm{P}<0.001)$, and further pairwise comparisons showed that the sorting of growing ovarian follicles was group $\mathrm{C}>$ group $\mathrm{A}>$ group $\mathrm{B}$. The sorting of mature ovarian follicles was group $\mathrm{C}>$ group $\mathrm{A}>$ group $\mathrm{B}$; the sorting of total ovarian follicles was group $\mathrm{C}>$ group $\mathrm{A}>$ group $\mathrm{B}$; the sorting of the ratio of mature ovarian follicles/total follicles was group $\mathrm{C}>$ group $\mathrm{A}>$ group $\mathrm{B}$; the sorting of the corpora lutea was group $\mathrm{C}>$ group $\mathrm{A}>$ group $\mathrm{B}$.

Table 1. Comparison of body weights among the three groups.

\begin{tabular}{|c|c|c|c|c|c|}
\hline Group & Cases & Original body weight & Body weight $10 \mathrm{w}$ later & Weight of ovaries & Ovarian index \\
\hline A & 10 & $34.7 \pm 1.16$ & $50.4 \pm 3.47$ & $0.0432 \pm 0.0078$ & $0.0009 \pm 0.0002$ \\
\hline C & 10 & $34.78 \pm 0.96$ & $55.95 \pm 11.11$ & $0.063 \pm 0.0112^{*}$ & $0.0012 \pm 0.0002^{*}$ \\
\hline B & 10 & $34.68 \pm 1.45$ & $48.91 \pm 3.05$ & $0.0302 \pm 0.0059^{*} \Delta$ & $0.0006 \pm 0.0001^{*} \Delta$ \\
\hline $\mathrm{F}$ & - & 0.06 & 2.145 & 46.767 & 23.217 \\
\hline$P$ & - & 0.98 & 0.112 & $<0.001$ & $<0.001$ \\
\hline
\end{tabular}

Note: ${ }^{*}$ Compared with group $A, P<0.05 ;{ }^{\Delta}$ compared with group $C, P<0.05$.

Table 2. Comparison of serum AMH, FSH, LH, and E24 among different groups.

\begin{tabular}{llllll}
\hline Group & Cases & AMH & FSH & LH & E2 \\
\hline A & 10 & $45.34 \pm 3.59$ & $28.05 \pm 2.72$ & $8.36 \pm 1.77$ & $32.08 \pm 3.89$ \\
\hline C & 10 & $50.72 \pm 5.14^{*}$ & $23.45 \pm 3.41^{*}$ & $6.84 \pm 0.51$ & $39.1 \pm 3.21^{*}$ \\
\hline
\end{tabular}

$\left.\begin{array}{lllllll}\hline \mathrm{B} & 10 & 43.24 & \pm 31.41 & \pm 11.05 & \pm 28.01 \\ & & 1.49^{\Delta} & 4.35^{*} \Delta & 3.55^{* \Delta} & 1.79^{*} \Delta\end{array}\right]$

Note: ${ }^{*}$ Compared with group $A, P<0.05 ;{ }^{\Delta}$ compared with group $B, P<0.05$.

Table 3. Comparison of ovarian follicles among different groups.

\begin{tabular}{|c|c|c|c|c|c|c|c|}
\hline Group & Cases & Growing ovarian follicles & Mature ovarian follicles & $\begin{array}{l}\text { Total } \\
\text { follicles }\end{array}$ & ovarian & $\begin{array}{l}\text { Mature ovarian follicles/ } \\
\text { total ovarian follicles }\end{array}$ & Corpus lutea \\
\hline A & 10 & $11.9 \pm 1.91$ & $1.8 \pm 0.79$ & $19.3 \pm 1.42$ & & $0.09 \pm 0.04$ & $6.7 \pm 0.82$ \\
\hline
\end{tabular}




\begin{tabular}{lllllll}
\hline $\mathrm{C}$ & 10 & $15 \pm 0.82^{*}$ & $3 \pm 0.67^{*}$ & $20.3 \pm 1.06$ & $0.15 \pm 0.03^{*}$ & $7.7 \pm 0.82$ \\
\hline $\mathrm{B}$ & 10 & $6.9 \pm 1.2^{*} \Delta$ & $1 \pm 0.82^{*} \Delta$ & $14.1 \pm 2.38^{*} \Delta$ & $0.07 \pm 0.06^{\Delta}$ & $3.2 \pm 1.69^{*} \Delta$ \\
\hline $\mathrm{F}$ & - & 85.616 & 17.633 & 31.45 & 8.244 & 31.646 \\
\hline $\mathrm{p}$ & - & $<0.001$ & $<0.001$ & $<0.001$ & $<0.001$ & $<0.001$ \\
\hline
\end{tabular}

Note: "Compared with group A, $\mathrm{P}<0.05 ;{ }^{\Delta}$ compared with group $\mathrm{C}, \mathrm{P}<0.05$.

\section{Discussion}

Tripterygium wilfordii is a woody climber with a bitter taste, cold nature, and an effective poison, but can promote blood circulation and remove meridian obstruction, dispel wind, eliminate dampness, kill worms and detoxify, and relieve swelling and pain. It has multiple bioactivities such as antitumor and anti-immune effects. TG is a group of bioactive substances extracted from $T$. wilfordii root fragments and is commonly used clinically to treat toxin and rheumatisminduced autoimmune diseases such as nephrotic syndrome and rheumatoid arthritis. However, its side effects have gained increasing attention, particularly those on the reproductive system. Short-term usage can cause menstrual cycle disorders in young women, and long-term usage may cause complete amenorrhea. According to previous studies, at doses greater than $800 \mathrm{mg}$, the incidence of amenorrhea was as high as $95 \%$.

Based on the above toxicities of TG towards the reproductive system, we selected this drug for modeling in this study to build a TG-OD model in mice [11] to further investigate the reproductive toxicities and mechanisms of TG, as well as TGinduced reproductive organ and ovarian damage and changes in sex hormones.

The dosage and medication timing when using TG to establish mouse models with follicular developmental disorder and ovarian dysfunctions are not standardized; in this study, we orally administered $0.35 \mathrm{~mL}$ of TG solution at $50 \mathrm{mg} /(\mathrm{kg} \bullet \mathrm{d})$ per day.

The results showed that after oral administration of $\mathrm{TG}$ solution, the mice exhibited poor general conditions and their estrous cycles were disturbed; the levels of FSH and $\mathrm{LH}$ increased, while the levels of AMH and E2 decreased. Mouse body weights and ovarian indices significantly decreased; the numbers of ovarian follicles, mature ovarian follicles, and corpus lutea were reduced, clearly indicating the reproductive toxicity of TG in mice. When endocrine disorders and ovarian follicular development disorders occur and the numbers of follicles and mature ovarian follicles are reduced, normal ovulation is affected. Thus, TG significantly disrupted mouse sex hormones and reproductive organs (ovaries).

TG brings new challenges to gynecological clinical practice for treating diseases. Therefore, predicting women's ovarian functions during treatment is an important research topic [12]. Currently, commonly used clinical methods include using estrogen and progestin for intervention during TG treatment to maintain the efficacies of TG, while also protecting ovary and other reproductive organ functions, thus avoiding or reducing
TG-induced premature ovarian failure and other side effects. However, long-term use of estrogen and progesterone also has some risks, such as migraine or frequent and abnormally severe headache, increased incidences of arterial and venous thrombosis, dementia, or breast cancer. Therefore, new, effective, and safe ovarian protective agents are urgently needed.

Gonadotropin-Releasing Hormone $(\mathrm{GnRH})$ is a 10-peptide hormone secreted by hypothalamic neurons. It stimulates the release of FSH and LH in the pituitary gland, promotes the secretion of estrogen and progesterone in the ovary, and promotes the development and maturation of follicles. GnRH analogs include gonadotropin-releasing hormone antagonists and GnRH agonists (GnRH-a) [13]. GnRH-a has been widely used in the clinic in recent years. GnRH agonist administration after all receptors, the pituitary is not sensitive to GnRH, FSH, LH secretion decreased significantly, ovarian hormone levels also decreased significantly, to appear artificial menopause, menopause, and to protect the ovarian function.

GnRH-a significantly protects against the toxic effects of chemotherapy drugs in the ovaries [14]. In a long-term clinical follow-up study of 240 children younger than 15 years who received chemotherapy to treat Hodgkin disease, only $13 \%$ of girls showed premature ovarian failure, while the percentage of boys with aspermia was as high as $83 \%$. These results indicate that ovarian functions before puberty are less affected by chemotherapeutic drugs; studies attempted to create a temporary preadolescence environment for women of childbearing age before chemotherapy to protect ovarian functions. As early as 1985, Ataya observed that long-acting GnRH-a TRI could protect against cyclophosphamide-induced ovarian damage in a rat model [15]. Subsequently, Yuan and Meirow confirmed the above conclusions in animal experiments [16], showing that GnRH-a could protect rat ovarian functions, save most of the primary follicles, and thus prevent quiescent follicles from damage caused by chemotherapeutic drugs $[17,18]$. Numerous clinical studies have confirmed that GnRH-a protects ovarian functions in patients from chemotherapy-induced damage $[19,20]$.

Based on the above findings, TG-induced reproductive toxicities can be prevented by GnRH agonists to protect ovarian functions. The TRI used in this study was a synthetic GnRH analog, the structure of which was modified by replacing the sixth L-amino acid (glycine) in the natural molecule with D-tryptophan. TRI has the same effects as GnRH; however, because of its longer plasma half-life, it exhibits stronger affinity to GnRH receptors. 
In this study, considering the effects of GnRH-a and that the mouse estrous cycle is 4-5 d, TRI was administered one week before this cycle and then co-administered with TG one week later. The results showed that the mice had lustrous fur, flexible activities, increased food and water intake, and body weight increase. FSH and $\mathrm{LH}$ were reduced, while $\mathrm{AMH}$ and $\mathrm{E} 2$ increased; the numbers of ovarian follicles and mature ovarian follicles increased, indicating that TRI significantly inhibited TG-induced reproductive toxicity, thus better protecting the development and maturation of growing follicles inside the ovaries.

TG can cause pathological conditions in the reproductive system of mice and significantly damage mouse hormone and reproductive organs. TRI protects against TG-induced gonad and ovarian dysfunctions in female mice. However, this study had a small sample size, and thus may not reflect overall changes; in addition, the administration timing and dosage require further analysis. Therefore, prospective, multi-center, and large-sample size studies are needed.

\section{Conflicts of Interest}

None

\section{References}

1. Liu TE, Zhang L, Wang S, Chen C, Zheng J. Tripterygium glycosides induce premature ovarian failure in rats by promoting p53 phosphorylation and activating the serine/ threonine kinase 11-p53-p21 signaling pathway. Exp Ther Med 2015; 10: 12-18.

2. Mans C, Pilny A. Use of GnRH-agonists for medical management of reproductive disorders in birds. Vet Clin North Am Exot Anim Pract 2014; 17: 23-33.

3. Charif SE, Inserra PI, Di Giorgio NP, Schmidt AR, LuxLantos V, Vitullo AD, Dorfman VB. Sequence analysis, tissue distribution and molecular physiology of the GnRH preprogonadotrophin in the South American plains vizcacha (Lagostomus maximus). Gen Comp Endocrinol 2016; 232: 174-184.

4. Badawy A, Elnashar A, El-Ashry M, Shahat M. Gonadotropin-releasing hormone agonists for prevention of chemotherapy-induced ovarian damage: prospective randomized study. Fertil Steril 2009; 91: 694-697.

5. Blumenfeld Z, Zur H, Dann EJ. Gonadotropin-releasing hormone agonist cotreatment during chemotherapy may increase pregnancy rate in survivors. Oncologist 2015; 20: 1283-1289.

6. Shen YW, Zhang XM, Lv M, Chen L, Qin TJ, Wang F, Yang J, Liu PJ, Yang J. Utility of gonadotropin-releasing hormone agonists for prevention of chemotherapy-induced ovarian damage in premenopausal women with breast cancer: a systematic review and meta-analysis. Onco Targets Ther 2015; 8: 3349-3359.

7. Lambertini M, Boni L, Michelotti A, Gamucci T, Scotto T, Gori S, Giordano M, Garrone O, Levaggi A, Poggio F, Giraudi S, Bighin C, Vecchio C, Sertoli MR, Pronzato P,
Del Mastro L. Ovarian suppression with triptorelin during adjuvant breast cancer chemotherapy and long-term ovarian function, pregnancies, and disease-free survival: a randomized clinical trial. JAMA 2015; 314: 2632-2640.

8. Del Mastro L, Boni L, Michelotti A, Gamucci T, Olmeo N, Gori S, Giordano M, Garrone O, Pronzato P, Bighin C, Levaggi A, Giraudi S, Cresti N, Magnolfi E, Scotto T, Vecchio C, Venturini M. Effect of the gonadotropinreleasing hormone analogue triptorelin on the occurrence of chemotherapy-induced early menopause in premenopausal women with breast cancer: a randomized trial. JAMA 2011; 306: 269-276.

9. Concannon PW, Temple M, Montanez A, Newton L. Effects of dose and duration of continuous GnRH-agonist treatment on induction of estrus in beagle dogs: competing and concurrent up-regulation and down-regulation of $\mathrm{LH}$ release. Theriogenology 2006; 66: 1488-1496.

10. Gris-Martínez JM, Trillo-Urrutia L, Gomez-Cabeza JJ, Encabo-Duro G. Protective effect of GnRH analogues on the reproductive capacity of women with neoplasia or autoimmune disease who require chemotherapy. Final results of a phase ii clinical trial. Med Clin (Barc) 2016; 146: 97-103.

11. Chen XY, Gu C, Ma M, Cong Q, Guo T, Ma D, Li B. A mouse model of premature ovarian insufficiency induced by tripterygium glycoside via subcutaneous injection. Int $\mathrm{J}$ Clin Exp Pathol 2013; 7: 144-151.

12. Fu Y, Zhao Z, Wu Y, Wu K, Xu X, Liu Y, Tong C. Therapeutic mechanisms of Tongmai Dasheng Tablet on tripterygium glycosides induced rat model for premature ovarian failure. J Ethnopharmacol 2012; 139: 26-33.

13. Hashem NM, El-Azrak KM, Nour El-Din AN, Taha TA, Salem MH. Effect of GnRH treatment on ovarian activity and reproductive performance of low-prolific Rahmani ewes. Theriogenology 2015; 83: 192-198.

14. Yang B, Shi W, Yang J, Liu H, Zhao H, Li X, Jiao S. Concurrent treatment with gonadotropin-releasing hormone agonists for chemotherapy-induced ovarian damage in premenopausal women with breast cancer: a meta-analysis of randomized controlled trials. Breast 2013; 22: 150-157.

15. Yüce MA, Balkanli Kaplan P, Gücer F, Doganay L, Altaner S, Canda T, Yardim T. Prevention of cyclophosphamideinduced ovarian damage by concomitant administration of GnRHa in mice: a dose-dependent relationship? Eur J Gynaecol Oncol 2004; 25: 628-631.

16. Meirow D, Assad G, Dor J, Rabinovici J. The GnRH antagonist cetrorelix reduces cyclophosphamide-induced ovarian follicular destruction in mice. Hum Reprod 2004; 19: 1294-1299.

17. Su J, Cheng J, Sun HX, Diao ZY, Zhen X, Yang J, Ding LJ, $\mathrm{Hu}$ YL. Tripterygium glycosides impairs the proliferation of granulosa cells and decreases the reproductive outcomes in female rats. Birth Defects Res B Dev Reprod Toxicol 2014; 101: 283-291.

18. Kanter M, Sapmaz-Metin M, Serez B. Effects of GnRHa on early embryonic development in mice receiving 
cyclophosphamide. Arch Gynecol Obstet 2016; 293: 203-209.

19. Park HJ, Koo YA, Im YH, Yoon BK, Choi D. GnRH agonist therapy to protect ovarian function in young Korean breast cancer patients. J Korean Med Sci 2010; 25: 110-116.

20. Li X, Kang X, Deng Q, Cai J, Wang Z. Combination of a GnRH agonist with an antagonist prevents flare-up effects and protects primordial ovarian follicles in the rat ovary from cisplatin-induced toxicity: a controlled experimental animal study. Reprod Biol Endocrinol 2013; 11: 16.

\section{*Correspondence to}

Ling Lv

Department of Gynecology and Obstetrics

The Second Affiliated Hospital of Zhejiang Chinese Medical University

PR China 